Asia Pac. J. Math. 2020 7:12

ASIA PACTFIC ACADEMIC

\title{
SECRET CODING METHOD ON STAR GRAPHS WITH GRAPH LABELING
}

\author{
G. UMA MAHESWARI ${ }^{1}$, SUZAN JABBAR OBAIYS ${ }^{2, *}$ \\ ${ }^{1}$ Department of Mathematics, Dhanalakshmi Srinivasan College of Engineering and Technology, \\ Mamallapuram, ECR, Chennai-603104 Tamil Nadu, India \\ ${ }^{2}$ School of Mathematical and Computer Science, Heriot-Watt University, Malaysia \\ Corresponding author: S.Obaiys@hw.ac.uk \\ Received Apr. 2, 2020
}

\begin{abstract}
AвSTRACT. In this paper we established a method of coding secret messages in the form of matrix by using two graphs. There are two illustrations with two different graphs super and sub super mean labeling and descriptions for the labeling is also provided. The function is Super Mean Labeling if $f(V) \cup\left\{f^{*}(e): e \in E(G)\right\}=\{1,2,3, \cdots, p+q\}$, and then the function is Sub Super Mean Labeling $f(V) \cup\left\{f^{*}(e): e \in E(G)\right\} \subset\{1,2,3, \cdots, p+q\}$.

2010 Mathematics Subject Classification. 05C78.
\end{abstract}

Key words and phrases. star graph; OBEF; EFOB; super mean labeling.

\section{INTRODUCTION}

An Engineer often finds that those real life problems that can be modeled into graphs small enough to be worked on by hand are also small enough to be solved by means other than graph theory. The high speed digital computer is one of the reasons for the recent growth of interest ein graph theory. This research work is devoted entirely to computational aspects of graph theory, including graph theoretic algorithms and samples of several tested computer programs for solving problems on graphs. whenever graph theory is applied to solving any practical problem (be it in electrical network analysis, in circuit layout, in data structures, in operations research or in social networks), it almost always leads to large graphs that are virtually impossible to analyze without the aid of the computer.

DOI: $10.28924 / A P J M / 7-12$

()2020 Asia Pacific Journal of Mathematics 


\section{Literature Review}

The concept of super mean labeling was introduced and studied by D. Ramya et al., ( [1]). Labeling two star graph using Mean Label was given by S. Somasundaram and R. Ponraj [2].The two star graph $K_{1, m} \cup K_{1, n}$ is a sub super mean graph which was proved by Uma Maheshwari et al., [3]. Coding through star graphs with super mean labeling is structured by Uma Maheswari et al., [4], [5] and coding with Fibonacci web is established by Uma Maheswari et al., [6].The super meanness property of the subdivision of the H-graph and slanting ladder was studied by R. Vasuki and A. Nagarajan [8] and super meanness of subdivision graph of $K_{1,3}$, some caterpillars and some duplicate graphs were studied by R. Vasuki and A. Nagarajan [9] and [10].

Motivated by these work, we worked on star graphs by applying super mean and sub super mean labeling and GMJ (Graph Message Jumbled) coding method and hence this paper.

\section{Definitions}

The original intelligible message is known as Plain text. The transformed message is known as Cipher text. An algorithm for transforming an intelligible message into one that is unintelligible by transposition and substitution methods is known as Cipher. A Caesar cipher shifts the alphabet and is therefore called a shift cipher. Each letter is replaced by the letter three positions further down the alphabet. Caesar used a key 3 for his communication and it is known as Caesar Cipher or Shift Cipher.

Super Mean Labeling Let $G$ be a $(\mathrm{p}, \mathrm{q})$ graph and $f: V(G) \rightarrow\{1,2,3, \cdots, p+q\}$ be an injection. For each edge $e=u v$, let

$$
f^{*}(e)=\left\{\begin{array}{cl}
\frac{f(u)+f(v)}{2} & \text { if } f(u)+f(v) \text { is even; } \\
\frac{f(u)+f(v)+1}{2} & \text { if } f(u)+f(v) \text { is odd }
\end{array}\right.
$$

then $\mathrm{f}$ is called Super Mean Labeling if $f(V) \cup\left\{f^{*}(e): e \in E(G)\right\}=\{1,2,3, \cdots, p+q\}$. A graph that admits a Super Mean Labeling is called a Super Mean Graph.

Sub Super Mean Labeling Let $G$ be a $(\mathrm{p}, \mathrm{q})$ graph and $f: V(G) \rightarrow\{1,2,3, \cdots, p+q\}$ be an injection. For each edge $e=u v$, let

$$
f^{*}(e)=\left\{\begin{array}{cl}
\frac{f(u)+f(v)}{2} & \text { if } f(u)+f(v) \quad \text { is even; } \\
\frac{f(u)+f(v)+1}{2} & \text { if } f(u)+f(v) \text { is odd }
\end{array}\right.
$$


then $f$ is called Sub Super Mean Labeling if $f(V) \cup\left\{f^{*}(e): e \in E(G)\right\} \subset\{1,2,3, \cdots, p+q\}$. A graph that admits a Sub Super Mean Labeling is called a Sub Super Mean Graph.

\subsection{A Method of Descriptions for three star super mean labeling.}

(1) Take $1, \frac{p+q+1}{2}$ and $p+q$ as $f(u), f(v)$ and $f(w)$ respectively. $f\left(u_{1}\right) \neq 2$, for the edge value becomes 2 when $f\left(u_{1}\right)=2$, but $f\left(v_{1}\right)=2$ is permitted as $f(v) \neq 1$.

(2) If $f\left(u_{1}\right)=3$, then $f\left(v_{1}\right)=4$ and if $f\left(u_{1}\right)=5$, then $f\left(v_{1}\right)=2$.

Assigning numbers on the first star is done first then the second and third stars are done side by side. An example for super mean labeling on a three star is given below:

Allot the smallest number omitted in the first star for the first pendant vertex of the second star. Immediately, allot the next least integer to the first pendant vertex of the third star. That is $f\left(v_{1}\right)$ and $f\left(w_{1}\right)$ must be allotted one after another. This process is maintained in allotting numbers to the pendant vertices of the second star and the third star. At times one has to continue in the same star for numbering two pendant vertices, one after another.

The figure representation is refereed by [4].

\section{Coding Method}

By assigning numbers to the 26 alphabets of English in a different manner, choosing a suitable labeled graph with a given clue mathematical or non-mathematical, finding the number in the graph for each letter of each word of the given message and presenting the letter codes in a unique way in some form, writing it as a horizontal string or in any other way and creating a picture with the codes after shuffling the order of the letters in order to increase the secrecy of the coded message is named as GMJ coding method. noindent GMJ code stands for:

(i) Graph Message Jumble code. A coding technique to communicate a Message through Graphs, Jumbling letters is named as GMJ code.

(ii) It also refers to the name of one of the Researchers (Gabriel Margaret Joan) who has conceived this method of coding.

At the same time, Graph theory has emerged as a worthwhile mathematical discipline in its own right. The theory is also intimately related to many branches of Mathematics including 
Group theory, Matrix theory, Numerical analysis, Probability, Topology and Combinatorics. By perusing through a number of text books on Graph theory, informations about history of Graph theory and its applications to various fields are gathered by inspiration of graph labeling along with coding technique this paper was developed.

\subsection{Numbering of alphabets: OBEF}

$\begin{array}{ccccccccccccc}13 & 14 & 12 & 15 & 11 & 16 & 10 & 17 & 9 & 18 & 8 & 19 & 7 \\ \text { A } & \text { B } & \text { C } & \text { D } & \text { E } & \text { F } & \text { G } & \text { H } & \text { I } & \text { J } & \text { K } & \text { L } & \text { M } \\ 20 & 6 & 21 & 5 & 22 & 4 & 23 & 3 & 24 & 2 & 25 & 1 & 26 \\ \text { N } & \text { O } & \text { P } & \text { Q } & \text { R } & \text { S } & \text { T } & \text { U } & \text { V } & \text { W } & \text { X } & \text { Y } & \text { Z }\end{array}$

The numbers 1 to 13 are allotted to the odd positioned alphabets moving backward from y to $\mathrm{w}$ to $\mathrm{a}$ and so on.

And the numbers 14 to 26 are allotted to the even positioned alphabets moving forward starting from $\mathrm{b}$ to $\mathrm{d}$ to $\mathrm{z}$ and so on.

This method is named as OBEF (odds backward, evens forward). We express the numbering of alphabets in terms of a function for encoding. For odd positioned alphabets the function $\mathrm{g}$ is given by,

$g(2 k+1)=(13-k)$ for $k=0,1,2, \cdots, 12$.

For odd positioned alphabets the function $\mathrm{g}$ is given by,

$g(2 k)=(13+k)$ for $k=1,3,5, \cdots, 13$.

For decoding we reverse the process.

The Greek letters $\delta, \eta$ and $\kappa$ are used to refer to the first, second and third star respectively. $t$, $v_{i}$ and $e_{i}$ denote the top vertex, the $i^{t h}$ pendant vertex and the $i^{t h}$ edge value in order. 


\section{Illustration 1:}

The transformation of text message into coded message

\begin{tabular}{|c|c|}
\hline plain text & cipher text \\
\hline Message & Get up at zero dark hundred. \\
\hline clue & Twinkling thrice and double the twins. \\
\hline Graph & $K_{1,4} \cup K_{1,4} \cup K_{1,4}$ \\
\hline Coding(word wise) & Letter Codes \\
\hline Get & $\delta\left(v_{4}\right) \eta\left(e_{1}\right) \kappa\left(e_{2}\right)$ \\
\hline up & $\delta\left(v_{1}\right) \kappa\left(e_{1}\right)$ \\
\hline at & $\eta\left(e_{2}\right) \kappa\left(e_{2}\right)$ \\
\hline zero & $\kappa\left(e_{4}\right) \eta\left(e_{1}\right) \kappa\left(v_{3}\right) \delta\left(e_{4}\right)$ \\
\hline dark & $\kappa\left(v_{1}\right) \eta\left(e_{2}\right) \kappa\left(v_{3}\right) \eta\left(v_{1}\right)$ \\
\hline hundred & $\eta\left(e_{4}\right) \delta\left(v_{1}\right) \eta\left(v_{4}\right) \kappa\left(v_{1}\right) \kappa\left(v_{3}\right) \eta\left(e_{1}\right) \kappa\left(v_{1}\right)$ \\
\hline Presenting the letter codes & $\begin{array}{l}\text { The functions } \delta, \eta \text { and } \kappa \text { are } \\
\text { removed to increase the secrecy } \\
\text { and presenting it in the horizontal string. }\end{array}$ \\
\hline Horizontal string & $\begin{array}{c}\left(v_{4}\right)\left(e_{1}\right)\left(e_{2}\right)(1,1)\left(v_{1}\right)\left(e_{1}\right) \\
(1,1)\left(e_{2}\right)\left(e_{2}\right)(1,1)\left(e_{4}\right)\left(e_{1}\right) \\
\left(v_{3}\right)\left(e_{4}\right)(1,1)\left(v_{1}\right)\left(e_{2}\right)\left(v_{3}\right) \\
\left(v_{1}\right)\left(e_{4}\right)\left(v_{1}\right)\left(v_{4}\right)\left(v_{1}\right)\left(v_{3}\right) \\
\left(e_{1}\right)\left(v_{1}\right)\end{array}$ \\
\hline Result: Matrix coding & $\left(\begin{array}{cccccc}\left(v_{4}\right) & \left(e_{1}\right) & \left(e_{2}\right) & (1,1) & \left(v_{1}\right) & \left(e_{1}\right) \\
(1,1) & \left(e_{2}\right) & \left(e_{2}\right) & (1,1) & \left(e_{4}\right) & \left(e_{1}\right) \\
\left(v_{3}\right) & \left(e_{4}\right) & (1,1) & \left(v_{1}\right) & \left(e_{2}\right) & \left(v_{3}\right) \\
\left(v_{1}\right) & \left(e_{4}\right) & \left(v_{1}\right) & \left(v_{4}\right) & \left(v_{1}\right) & \left(v_{3}\right) \\
\left(e_{1}\right) & \left(v_{1}\right) & (0,0) & (0,0) & (0,0) & (0,0)\end{array}\right)$ \\
\hline
\end{tabular}


4. A Method Descriptions for Three Star Sub Super Mean Labeling

If $G=K_{1, \ell} \cup K_{1, m} \cup K_{1, n}$ admits $\operatorname{SSML}\left(V_{4}, E_{2}\right)$ with $2 \leq \ell$, or $2 \leq(n-m)+\ell$ and $g=$ $\left[\frac{n+\ell-m}{2}\right]$, then

(A) If $p+q=2 \ell+2 m+2 n+3=4 J+3, J=\ell+K, K=\left[\frac{n+m-\ell}{2}\right]$, the graph is a $(g-1) \mathrm{RO}, g \mathrm{RO}$ and $(g+1) \mathrm{RO}$ graph according as both FEIO, SEIO accommodated, any one is accommodated and both not accommodated respectively.

(B) If $(p+q)=2 \ell+2 m+2 n+3>4 J+3$, then the graph is $g \mathrm{RO},(g+1) \mathrm{RO}$ and $(g+2)$ $\mathrm{RO}$ respectively as stated in $(\mathrm{A})$.

The figure representation is referred by [7]

\subsection{Numbering of alphabets: EFOB}

$\begin{array}{ccccccccccccc}26 & 1 & 25 & 2 & 24 & 3 & 23 & 4 & 22 & 5 & 21 & 6 & 20 \\ \text { A } & \text { B } & \text { C } & \text { D } & \text { E } & \text { F } & \text { G } & \text { H } & \text { I } & \text { J } & \text { K } & \text { L } & \text { M } \\ 7 & 19 & 8 & 18 & 9 & 17 & 10 & 16 & 11 & 15 & 12 & 14 & 13 \\ \text { N } & \text { O } & \text { P } & \text { Q } & \text { R } & \text { S } & \text { T } & \text { U } & \text { V } & \text { W } & \text { X } & \text { Y } & \text { Z }\end{array}$

The numbers 1 to 13 are allotted to the even positioned alphabets moving forward from $b$ to $d$ to $\mathrm{z}$ and so on. And the numbers 14 to 26 are allotted to the odd positioned alphabets moving backward starting from $\mathrm{y}$ to $\mathrm{w}$ to a and so on. This method is named as EFOB (evens forward, odds backward).

We express the numbering of alphabets in terms of a function for encoding. For odd positioned alphabets the function $\mathrm{g}$ is given by,

$$
g(2 k+1)=(26-k) \text { for } k=0,1,2, \cdots, 12 .
$$

For odd positioned alphabets the function $\mathrm{g}$ is given by,

$$
g(2 k)=(13) \text { for } k=1,3,5, \cdots, 13 .
$$

For decoding we reverse the process.

4.2. Coding a letter: Perhaps of all the creations of man, language is the most astonishing. The first business of language is simply to be transparent. With the dynamic living of the people and the growth of civilization, the need to replace transparency by secrecy increases. In any area, when messages and information need to be communicated only to a group of people or to an individual, one is not able to use a common language. The communication 
becomes very much limited between the sender and the receiver and not to be understood by others.

A triplet is used to code a letter. Corresponding to the letter in the message, the number is taken and it is located in the graph. If it is in the second star, fifth pendant vertex, then the required triplet is $(2,5,0)$. The top vertices of the three star are denoted by $(1,1,1),(2,2,2)$ and $(3,3,3)$. Cryptography is the science containing methods to transform an intellible message into one that it is unintelligible and transforming the message back to its original form. The oldest types of ciphers (algorithm) was developed by Julius Caesar.

Illustration 2: The transformation of text message into coded message

\begin{tabular}{|c|c|}
\hline plain text & cipher text \\
\hline Message & Cherished irons near RM (Rockey Mountain). \\
\hline clue & Twinkling thrice and double the twins. \\
\hline Graph & $K_{1,4} \cup K_{1,4} \cup K_{1,4}$. \\
\hline Coding(word wise) & Letter Codes \\
\hline Cherished & $\begin{array}{c}(3,3,0)(1,0,2)(3,0,2)(2,2,0)(3,0,1) \\
(2,4,0)(1,0,2)(3,0,2)(1,0,1)\end{array}$ \\
\hline irons & $(3,0,1)(2,2,0)(2,2,2)(1,2,0)(2,4,0)$ \\
\hline near & $(1,2,0)(3,0,2) 2(2,3,0)(2,2,0)$ \\
\hline BR & $(2,2,0)(3,4,0)$ \\
\hline Presenting the letter codes & $\begin{array}{l}\text { The single numbers representing letters which } \\
\text { are obtained as explained in coding } \\
\text { a letter are just written } \\
\text { in a horizontal string }\end{array}$ \\
\hline Horizontal string & 635446352446363510447 \\
\hline Result: Matrix coding & $\left(\begin{array}{ccccccc}6 & 3 & 5 & 4 & 4 & 6 & 3 \\
5 & 2 & 4 & 4 & 6 & 3 & 6 \\
3 & 5 & 10 & 4 & 4 & 7 & 0\end{array}\right)$ \\
\hline
\end{tabular}




\section{Algorithm}

- step 1: Guess the graph.

- step 2: label the graph.

- step 3: Split the alphabets.

- step 4: Define coding method of the text message.

- step 5: Write the original message.

- step 6: Apply letter codes word wise.

- step 7: Representing codes into Horizontal string .

- step 8: Representing string into matrix form.

The communicator has to pass these steps to the decoder:

- clue for graph

- Clue for numbering of alphabets without abbreviation.

- string or matrix

\section{Conclusion and Future Work:}

In this paper we have settled the star graph is a super and sub super mean labeling. We illustrated how the text message is converted to matrix code. Clues for numbering of alphabets are newly introduced in the development of a coding technique. In this research article we have shown how the secret message is transformed into coded message using two and three star graph. In our future work we would like to apply labeling for five star graphs and develop coding techniques for them. 


\section{REFERENCES}

[1] P. Jayenthi, D. Ramya and P. Thangavelu, On Super Mean Labeling of Some Graphs, SUT J. Math. 46 (1) (2010), 53-66.

[2] S. Somasundaram and R. Ponraj, Mean labeling of graphs, Nat. Acad. Sci. Lett. 26 (2003), 210 - 213.

[3] G. Uma Maheswari, G. Margaret Joan Jebarani and V. Balaji, On Similar Super Mean Labeling for two star graph, Asian J. Math. Computer Res. 21 (2) 2017, 53 - 62.

[4] G. Uma Maheswari, G. Margaret Joan Jebarani and V. Balaji, Coding Through a Two Star and Super Mean Labeling. (Springer - Applied Mathematics and Scientific Computing, volume 2, 2017).

[5] G. Uma Maheswari, G. Margaret Joan Jebarani and V. Balaji, GMJ Coding Through a Three Star and Super Mean Labeling, Amer. Int. J. Res. Sci. Technol. Eng. Math. Accepted.

[6] [1]G.U. Maheswari, G.M.J. Jebarani, V. Balaji, Coding techniques through Fibonacci webs, difference cordial labeling and GMJ code method, J. Phys.: Conf. Ser. 1139 (2018) 012077.

[7] G. Uma Maheswari, G. Margaret Joan Jebarani and V. Balaji, Sub Super Mean Labeling on a General Three Star Graph, Int. J. Math. Appl. 6(1) (2018), 147 - 153.

[8] R. Vasuki and A. Nagarajan, On the Construction of New Classes of Super Mean Graphs, J. Discr. Math. Sci. Cryptogr. 13 (3) (2010), 277 - 290.

[9] R. Vasuki, P. Sugirtha and J. Venkateshwari, Super Mean Labeling of some subdivision graphs, Kragujerac J. Math. 41 (3) (2017), 179 - 201.

[10] S. K. Vaidya and L. Bijikumar, Some New Families of Mean Graphs, J. Math. Res. 2 (3) (2010), 169 - 176. 